\title{
Artículo Original: Efectividad de Trastuzumab en combina- ción con terapia sistémica en pacientes con cáncer de mama avanzados o metastásico HER2 positivos. Experiencia del Hospital México, Costa Rica
}

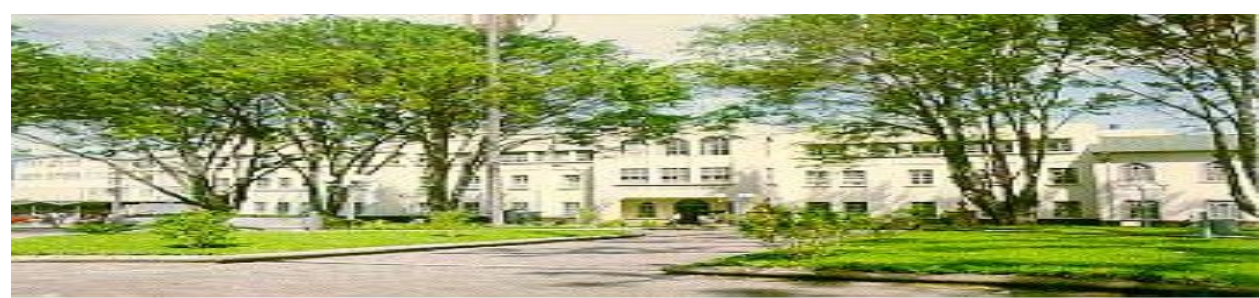

Haspital San quan de Dios. San Yosé, Costa Rica. Fundada en 1845

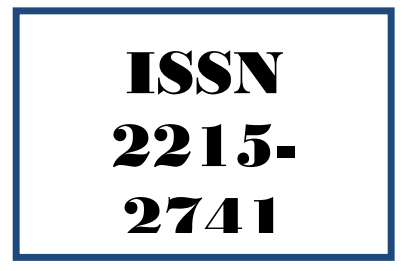

Recibido:

$18 / 07 / 2017$

Aceptado:

$15 / 08 / 2017$

Juan P. Molina ${ }^{1}$

Denis U. Landaverde ${ }^{2}$

\footnotetext{
${ }^{1}$ Servicio de Oncología del Hospital México-Caja Costarricense de Seguro Social. jpmolina@,ccss.sa.cr

${ }^{2}$ Profesor de la Escuela de Medicina Universidad de Costa Rica. denislandaverde@gmail.com
}

\section{RESUMEN}

Introducción: Este estudio reporta la efectividad y seguridad del uso de trastuzumab en combinación con terapia sistémica en el tratamiento de pacientes con cáncer de mama localmente avanzado o metastásico, HER2 positivos. Se muestra la experiencia de los primeros cinco años del uso de este fármaco en el Hospital México, San José Costa Rica.

Materiales y Métodos: Se trata de un estudio retrospectivo, observacional de pacientes con cáncer de mama HER2 positivos, con enfermedad metastásica o localmente avanzada irresecable, se analizaron los casos de enero de 2006 a diciembre 2011. El estado HER2 fue analizado mediante inmunohistoquímica, y en caso de duda con Hibridación in situ flurescente $(F I S H)$. Se reporta la efectividad del trastuzumab en combinación con quimioterapia u hormonoterapia. Así mismo se incluyó aquellos pacientes con metástasis cerebrales y que durante la adyuvancia recibieron trastuzumab.

Resultados: Un total de 301 expedientes fueron encontrados pero solo 39 cumplieron los criterios de inclusión. 28 pacientes $(65 \%)$ recibieron trastuzumab como primera línea. 29 (67\%) recibieron trastuzumab en combinación con paclitaxel (tanto en primera como líneas subsecuentes). La mediana de progresión a la primera línea con trastuzumab fue de 6.56 meses (C195\% 3.57-9.65 meses). La mediana de supervivencia reportada con quimioterapia más trastuzumab fue de 33.5 meses (CI 95\% 22.144.8 meses). Se reportaron 3 eventos cardiacos sintomáticos que requirieron suspensión temporal del trastuzumab. Se encontró una tendencia a una mejor superviviencia en pacientes con receptores de estrógeno positivos pero ésta no fue estadísticamente significativa $(\mathrm{p}=0.066)$. 
Conclusiones: En este estudio de la vida real se encuentra que trastuzumab en combinación con quimioterapia se asocia con una mediana a la progresion y superviviencia general muy similar a la reportada en los estudios de registro del trastuzumab. El trastuzumab tiene un perfil de cardio-toxicidad muy favorable en esta población Latinoamericana.

\section{PALABRAS CLAVE}

Cáncer de Mama, Metástasis, HER2, Trastuzumab, Costa Rica.

\section{ABSTRACT}

Introduction: We report the effectiveness and safety profile of trastuzumab in combination with systemic treatments in an unselected group of patients with unresectable locally advanced and/or metastatic HER2 positive breast cancer. This is a single center experience of the initial five years' experience of trastuzumab in Hospital MexicoCosta Rica.

Materials and Methods: This is a retrospective and observational study of patients with HER2 positive locally advanced or metastatic breast cancer, between Jan 2006 and Dec 2011. HER2 status was confirmed by imnunohistochemistry $(3+)$ or fluorescence in situ hybridization (FISH). We report here the data from the addition of trastuzumab to any systemic treatment used, including anti-hormonal drugs. We included patients with brain metastases, as well as patients who received trastuzumab in the adjuvancy.

Results: A total of 301 records were found but only 39 of them fulfill all the inclusion criteria. 28 patients $(65 \%)$ received trastuzumab as first line regimen. 29 patients $(67 \%)$ received trastuzumab plus paclitaxel. The median time to progression with first line chemotherapy regimen with trastuzumab was 6.56 months (CI 95\% 3.57-9.65 months). The median survival reported with chemotherapy plus trastuzumab was 33.5 months (CI 95\% 22.1- 44.8 months). Three symptomatic cardiac events were reported, and this cause a temporary withdrawal of the trastuzumab administration. There was a tendency of superior in median overall survival in patients with positive estrogen receptor status but it was not statistically significant (log rank 0.066).

Conclusion: In this group of unselected patients attended in our Institution, the use chemotherapy plus trastuzumab was associated with a median time to progression and overall survival very similar to the experience reported in the pivotal trials of trastuzumab. And also de cardio-toxicity was mild in this Latin American population

\section{KEY WORDS}

Breast Cancer, Metastasis, HER2, Trastuzumab, Costa Rica.

\section{INTRODUCCIÓN}

La proteína codificada por el gen HER2/neu (se encuentra sobreexpresada en cerca del $25 \%$ de las pacientes con cáncer de mama y es el oncogén con mayor incidencia identificado en esta neoplasia (1). La presencia de esta característica se asocia a un fenotipo clínico agresivo que incluye tumores de alto grado, con mayores tasas de crecimiento, aparición de metástasis tempranamente, así como periodos libres de enfermedad y sobrevida general menores ${ }^{(2-6)}$.

El trastuzumab es un anticuerpo monoclonal dirigido contra el dominio extracelular del receptor HER2 que ha demostrado, luego de más de 15 años de experimentación en seres humanos, un beneficio notorio en los tratamientos adyuvantes y de enfermedad avanzada en esta condición ${ }^{(7,8)}$. Ha sido evaluado para su uso como monoterapia o terapia de combinación con tratamientos hormonales, diversos quimioterapéuticos e incluso otros tratamientos contra la vía del HER2 ${ }^{(9-11)}$.

El uso de terapia dirigida contra el receptor HER2 se considera actualmente el estándar de cuido en esta enfermedad ${ }^{(12)}$. Desde el año 2006 el trastuzumab está disponible en el sistema de seguridad social de Costa Rica (Caja Costarricense de Seguridad Social) como parte de los tratamientos ofrecidos a las pacientes portadoras de cáncer de mama metastásico HER2 positivo. 


\section{MATERIALES Y MÉTODOS}

Este protocolo de investigación fue aprobado por el Comité Local de Bioética (CLOBI) del Hospital México según resolución CLOBI HM 007-2014. Esta investigación no contó con patrocinio externo ni contó con protocolos de investigación paralelos.

El objetivo principal de este trabajo fue determinar la efectividad y la seguridad del uso de trastuzumab en la práctica clínica diaria en las pacientes tratadas por cáncer de mama avanzado HER2 positivo. Como objetivos secundarios se intentó determinar la mediana de supervivencia global en la muestra, los sitios más frecuentes de metástasis y si existen diferencias clínicas según el estado de sobreexpresión de receptores hormonales en el tumor.

Para la identificación de los casos se procedió a la revisión manual de los expedientes clínicos y registros electrónicos disponibles de todas las pacientes a quienes se les había solicitado aprobación de uso de trastuzumab entre enero de 2006 y diciembre de 2011.

Se incluyó los datos de todas las pacientes con diagnóstico de cáncer de mama irresecable o metastásico, con confirmación por inmunohistoquímica o por medio de FISH (fluorecense in situ hidridization), quienes fueron tratadas con trastuzumab con intención de manejo paliativo. Se incluyó la información de todas las pacientes femeninas, mayores de 18 años y en cualquier estado funcional al momento de prescribir el medicamento. Se incluyó la información de pacientes con antecedente de metástasis a sistema nervioso central ya tratadas y pacientes con exposición previa a trastuzumab como parte de un tratamiento adyuvante.

La supervivencia libre de progresión se calculó desde el momento en que se adicionaba el trastuzumab a cualquier tratamiento sistémico ofrecido con intención paliativa, hasta que el clínico realizara una modificación del esquema por documentarse progresión, toxicidad o se produjera el fallecimiento.
Se realizó un análisis de correlación de la probabilidad de sobrevida libre de progresión y sobrevida global mediante el método de KaplanMeier. También se intentó determinar por este medio la correlación pronóstica según el estado de expresión de receptores hormonales. Los cálculos estadísticos se realizaron utilizando el software Microsoft Excel 2010 y SPSS versión 13.0 definiendo intervalos de confianza de $95 \%$ y determinando la significancia de los datos mediante el método Mantel-Cox (test log rank).

\section{RESULTADOS}

En los registros de administración de medicamentos en nuestro centro se encontró que se le había aplicado el fármaco a 221 pacientes en este periodo y se le había solicitado su aprobación a 88 pacientes más. Sin embargo únicamente a 43 pacientes se les había solicitado el fármaco como parte de un tratamiento.

La histología más frecuente fue el carcinoma ductal infiltrante de alto grado en $54 \%$, seguido del carcinoma ductal infiltrante de grado II en $28 \%$. Se especificó carcinoma lobulillar invasor en únicamente una paciente. Se encontró un fenotipo HER2 enriquecido en $67 \%$ de los pacientes y sobreexpresión adicional de receptores de estrógeno, es decir fenotipo Luminal-HER 2 conducido en $33 \%$

La confirmación de sobreexpresión de receptores HER2 se hizo en $95 \%$ de los casos a través de inmunohistoquímica y en dos casos mediante FISH. Durante este periodo y particularmente en los primeros años de su uso, el médico prescriptor desconocía el resultado de la positividad del estado de positividad de HER2 al momento de decidir la primera línea de tratamiento metastásico, hasta en un $54 \%$ de los casos.

Un $92 \%$ de los pacientes habían recibido tratamiento previo con quimioterapia y un $57 \%$ ya habían sido expuestas previamente a trastuzumab con intención neoadyuvante o adyuvante. Particularmente la quimioterapia neoadyuvante se había utilizado en $26 \%$ de las pacientes, pero en únicamente la mitad se utilizó trastuzumab con esa intención y solamente en una de las pacientes 
se había obtenido una respuesta patológica completa.

Al momento del diagnóstico un $18 \%$ de las pacientes presentaban enfermedad recurrente o metastásica. Los sitios más frecuentes de aparición de metástasis fueron el hueso (29\%), seguido de pulmón (16\%), hígado (14\%), SNC (10\%) y $8 \%$ correspondieron a recurrencias locales.

La mediana del periodo libre de progresión (PLP) en la muestra fue de 19,3 meses (rango 054.4 meses) y la mediana de duración entre el momento del diagnóstico hasta la primera aplicación de trastuzumab con intención paliativa fue de 24,6 meses. (Gráfico No.2)

Durante el periodo 2006-2011, la incorporación del trastuzumab al arsenal terapéutico de la C.C.S.S fue gradual, e inicialmente algunas pacientes lograron obtener el fármaco como parte de un tratamiento sistémico más allá de la primera línea.

Se utilizó la combinación de trastuzumab-paclitaxel en $67 \%$ de los casos, seguido de trastuzumab-capecitabina en $18 \%$ y en algunos casos en combinación con lapatinib, anastrazol y tamoxifeno. Ninguna paciente recibió trastuzumab en combinación con antraciclinas.

A pesar de que algunos pacientes lograban recibir diversos esquemas y drogas como parte del tratamiento paliativo; algunas de las cuales incluyeron estrategias de bloqueo dual de la vía del HER2 o la incorporación de drogas trastuzumab-emtansine, la mayoría de pacientes recibieron solamente una o dos líneas de tratamiento.

La mediana del periodo libre de progresión combinado posterior a la adición de trastuzumab a una línea de tratamiento sistémico fue de 6,56 meses (IC 95\% 3,57 a 9,65 meses). En el subgrupo de pacientes que fueron tratadas con trastuzumab-paclitaxel fue de 6,67 meses (IC 95\% 3,34 a 10,0 meses)

La mediana de supervivencia, calculada a partir de la primera dosis de administración de trastuzumab, fue de 33,5 meses (IC 95\% 22,1-44,8 meses).

La sobreexpresión de receptores hormonales se asoció aunque sin lograr significancia estadística a una mejoría en la sobrevida global (Log Rank: $\mathrm{p}=0.066)$ (Figura No.4)

Se encontró registros de tres pacientes que desarrollaron toxicidad cardiaca. Dos presentaron toxicidad grado III y otra grado I según los criterios del NCI. La primera paciente tenia al momento 61 años, no presentaba comorbilidades, recibió antraciclinas en la adyuvancia, recibió el trastuzumab en combinación con paclitaxel y presentó insuficiencia cardiaca leve así como caída de la $\mathrm{FE}>20 \%$, y luego de una interrupción temporal se logró reintroducción de la droga. La otra paciente que desarrollo toxicidad grado III, tenía al momento 48 años, no tenía comorbilidades, había sido altamente pre-tratada y tenía una dosis acumulada de $900 \mathrm{mg} / \mathrm{m} 2$ de epirrubicina, el episodio de insuficiencia cardiaca lo presentó luego de una re-exposición a trastuzumab más paclitaxel después de recibir 4 ciclos con intención paliativa de FEC.

La tercera paciente tenía en ese momento 68 años, tampoco tenía comorbilidades, también había recibido antraciclinas como tratamiento adyuvante y ameritó una interrupción temporal del medicamento por caída de la $\mathrm{FE}>20 \%$ aunque sin traducción clínica.

\section{DISCUSIÓN}

La mediana del periodo libre de progresión obtenida en esta investigación fue de 6,56 meses (IC 95\% 3.57 a 9.65). El resultado anterior es muy similar al obtenido al estudio pivotal publicado por Slamon, en el cual la adición de trastuzumab al grupo tratado con quimioterapia basada en antraciclinas o taxanos obtuvo un periodo libre de progresión de 7.4 meses. En dicho estudio la sobrevida libre de progresión en el subgrupo de pacientes que recibieron específicamente paclitaxel más trastuzumab fue de 6.9 meses lo cual se acerca mucho al resultado obtenido en esta investigación ${ }^{(2)}$.

El estudio HERMINE fue un estudio fármacoepidemiológico retrospectivo y prospectivo, conducido en Francia para evaluar el resultado del uso de trastuzumab en 623 pacientes con características basales son similares a nuestra muestra. Al comparar los datos del periodo libre de progresión los resultados en esa muestra fueron mejores. En el estudio HERMINE el tiempo para primera progresión fue de 10.3 
meses, 9.0 meses para la segunda progresión y 6.3 meses para la tercera ${ }^{(13)}$. Debe tenerse presente, que para el momento de realizarse el estudio HERMINE el uso continuado de trastuzumab más allá de la progresión era una práctica común ${ }^{(14-16)}$.

La mediana de sobrevida global posterior al inicio de trastuzumab con intención paliativa fue de 33.5 meses (IC 95\%: 22.1-44.8). Lo cual fue ligeramente mayor a lo descrito en los estudios HERMINE ( $m=30,3$ meses) y el estudio pivotal $(\mathrm{m}=25.1$ meses $)$. La mediana de supervivencia global fue ligeramente mayor a lo reportado tanto en el estudio pivotal como en el HERMINE. Dicho resultado podría estar influenciado por una alta representación en la muestra de pacientes con sobrevidas muy prolongadas.

El efecto de la sobreexpresión de receptores hormonales en el comportamiento biológico de las pacientes con amplificación de gen HER2 es objeto de intenso estudio y de controversia ${ }^{(17-19)}$. En España y China la prevalencia de tumores luminales HER2 conducidos es cercana al $12 \%$ en tanto que los tumores HER2 enriquecidos representan cerca de $7 \%$ de todos los casos de $\mathrm{CM}$ y en otros reportes la representación de ambos subtipos es similar ${ }^{(20-22)}$.

En nuestro caso, la totalidad de pacientes descritas corresponden a casos de enfermedad avanzada o recurrente, y aunque un $64 \%$ de las pacientes corresponden al subtipo HER2 enriquecido, no se puede inferir por el tipo de estudio y lo limitado de la muestra que este inmunofenotipo representa una biología más agresiva o asociado a mayor recurrencia ${ }^{(22,23)}$. En esta investigación y a pesar de una clara separación de las curvas, no se logró demostrar un beneficio con significancia estadística de mejoría en la sobrevida global en los pacientes que presentaban expresión de receptores hormonales $(p=0.066)$.

En congruencia con los resultados de estudios epidemiológicos, las metástasis óseas fueron el sitio metastásico de más frecuente de presentación. En nuestra investigación, la prevalencia fue de $50 \%$, similar a lo descrito por Kennecke y cols quienes encontraron una frecuencia de $65 \%$ en los tumores luminalesHER2 y $59,6 \%$ en los tumores HER2 enriquecidos, lo anterior en una población que no había sido tratada con trastuzumab de forma adyuvante ${ }^{(24)}$. Las metástasis cerebrales constituyeron un $13 \%$ del total de sitios metastásicos encontrados en la población estudiada. Esta cifra es muy cercana a lo reportado por Burstein en una cohorte de 518 pacientes portadoras de carcinoma de mama metastásico HER2 enriquecido ${ }^{(25)}$. Al igual que lo demostrado en estas investigaciones, las metástasis a SNC se presentaron generalmente posterior a haberse documentado progresión en sitios periféricos.

Uno de los aspectos que contribuyen al diagnóstico más tardío de esta presentación es la menor tasa de realización de estudios de imágenes rutinarios y por ende un sesgo de nodetección temprana. Hasta el momento no existe evidencia concluyente de que el uso de trastuzumab acelere o prevenga la progresión de la enfermedad en SNC. Se postula que la frecuencia de metástasis aislada a SNC es consecuencia de varios factores clínicos y biológicos.

Primeramente la mejoría en el control de la enfermedad periférica y el aumento de la supervivencia podrían dejar a las pacientes vulnerables a la progresión en de una enfermedad oculta y subclínica ${ }^{(26,27)}$. En un segundo término, se considera al SNC un sitio santuario en donde las concentraciones de la droga en LCR en condiciones normales son demasiado bajas ${ }^{(28)}$. Las metástasis pulmonares al igual a lo reportado por Kennecke, fueron el segundo sitio más frecuente de presentación y en nuestro caso fue la localización más frecuentemente en que las pacientes debutaron como enfermedad metastásica ${ }^{(24)}$.

El estudio Retreatment after HErceptin Adjuvant (RHEA) fue un estudio prospectivo diseñado para evaluar la eficacia $y$ seguridad de trastuzumab en pacientes portadoras de CMM quienes previamente habían recibido el medicamento de forma adyuvante (en nuestra muestra el uso de trastuzumab en adyuvancia fue de $65 \%$ ). En ese se demostró que aunque la mayoría de los pacientes presentan disminución $<10 \%$ de la FE durante el periodo evaluado, cerca de un $15 \%$ presentan disminución de la $\mathrm{FE}$ $>10 \%$. Sin embargo la frecuencia de suspensión o retiro del medicamento por desarrollo de 
insuficiencia cardiaca es un fenómeno infrecuente $^{(28)}$.

En esta investigación se documentaron dos episodios de toxicidad cardiaca grado III y una grado I, es decir cerca de $8 \%$ de todas la pacientes expuestas a la droga, lo cual ubica nuestra muestra en una posición intermedia entre estudios que reportan una tasa de eventos cardiacos $<2 \%$ como el estudio RHEA y los reportes que revelan una tasa mayor del $10 \%$ como los reportes de farmacovigilancia en España o el estudio pivotal de Slamon ${ }^{(2,29)}$. Debe considerarse el hecho de que en nuestra muestra el número promedio de estudios ecocardiografícos realizados en la sobrevida posterior al inicio de trastuzumab fue de solo 2.64, por lo que probablemente existe un sesgo de no detección.

Por la naturaleza de este estudio de la vida real, se encontró varios problemas que pueden alterara la superviviencia de las pacientes, como falta inicial de una biopsia confirmatoria, inicios tardíos de quimioterapia, la realización tardía de estudios confirmatorios del estado metastásico y de estadiaje. Se detectaron dos casos en los cuales las pacientes habían iniciado quimioterapia adyuvante cerca de las 10 semanas desde el postoperatorio y todavía no se habían realizado ultrasonidos de abdomen $u$ otras técnicas de imagen, y que en el momento de su realización mostraron metástasis hepáticas.

En el caso de pacientes con sospecha de enfermedad metastásica también se detectó un retraso de hasta varios meses en la realización de estudios como tomografías, ultrasonidos y gammagrafías. Esto implica el riesgo de recibir un tratamiento tóxico, en ocasiones de muy alto costo y con una eficacia subóptima.

La toxicidad cardiaca es el principal riesgo del uso de trastuzumab (29,30). El monitoreo ecocardiográfico o mediante técnicas de medicina nuclear como MUGA permiten detectar la disfunción sistólica en una fase presintomática y podría disminuir el desarrollo de ICC NYHA III y IV ${ }^{(31-33)}$. En el presente estudio sin embargo se evidenció que el número de estudios ecocardiográficos se realizaban con una frecuencia promedio de uno cada 11 meses, por lo que es evidente que se debe mejorar la logística para aumentar la cantidad de estudios practicados y de esta forma la tasa de detección de toxicidad subclínica.

Se encontraron entre los expedientes analizados dos pacientes diagnosticados con cáncer de mama metastásico HER2 positivo que fallecieron en espera de aprobación de trastuzumab entre los años 2006 y 2007. En el año 2010 se diagnosticaron tres pacientes con metástasis óseas como único sitio metastásico y se postergó el inicio de trastuzumab hasta el desarrollo de enfermedad visceral. Desde el año 2010 hasta el 2011 se encontró registro de al menos 5 solicitudes de aprobación de lapatinib, de las cuales solamente 2 fueron aprobadas, y ambas pacientes iniciaron el medicamento después de 3 meses de haberse documentado la progresión.

Es claro que el costo de las terapias dirigidas es sumamente elevado, $y$ es innegable el establecimiento de controles especiales para garantizar el mayor beneficio clínico con la inversión económica realizada. Sin embargo, este tipo de retrasos implican un deterioro en el estado funcional de los pacientes, aumento del riesgo de progresión en otros sitios y pueden propiciar la re-exposición a tratamientos citotóxicos con los cuales ha ocurrido progresión en un intento heroico por controlar la enfermedad.

Es necesario revisar los mecanismos de aprobación institucional de medicamentos en esta enfermedad, establecer lineamientos claros de los criterios para aprobación y discutir a la luz de evidencia científica la revisión continua de tales criterios.

\section{CONCLUSIONES}

Durante los primeros cinco años de uso institucional de trastuzumab en pacientes portadoras de cáncer de mama metastásico HER2 positivo, el periodo libre de progresión del primer esquema de tratamiento sistémico que incorporó trastuzumab así como la supervivencia general son comparables con lo obtenido en los estudios de registro iniciales de la droga y estudios en poblaciones no seleccionadas atendidas en la práctica clínica rutinaria en tratamientos de primera línea. Además el perfil de toxicidad de trastuzumab fue bajo, y bien 
tolerado, también similar a lo reportado en los estudios pivotales de registro. Éste es el primer estudio reportado en Costa Rica y en Centro América del cual se tiene conocimiento a la fecha mostrando que trastuzumab en combinación con terapia sistémica es efectiva y segura en ésta población Latinoamericana.

\section{RECOMENDACIONES}

Times New Roman 10. Justificado. Separadas por punto y aparte.

\section{BIBLIOGRAFÍA}

1. Niño LAG, Garavito AÁ, Jaramillo CE, Velásquez SJ, Corcho RDS, Bernal BHA. Cáncer de mama: HER2/neu, métodos diagnósticos y consideraciones clínicas. Rev Colomb Cancerol. 2007;11(1):40-57.

2. Slamon DJ, Leyland-Jones B, Shak S, Fuchs H, Paton V, Bajamonde A, et al. Use of chemotherapy plus a monoclonal antibody against HER2 for metastatic breast cancer that overexpresses HER2. N Engl J Med. 2001;344(11):783-92.

3. Holleczek B, Jansen L, Brenner H. Breast cancer survival in Germany: a population-based high resolution study from saarland. PloS one. 2013;8(7):e70680.

4. Burstein HJ, Lieberman G, Slamon DJ, Winer EP, Klein P. Isolated central nervous system metastases in patients with HER2-overexpressing advanced breast cancer treated with first-line trastuzumab-based therapy. Ann Oncol. 2005 Nov;16(11):1772-7.

5. McCormack VA, Joffe $M$, van den Berg E, Broeze N, dos Santos Silva I, Romieu I, et al. Breast cancer receptor status and stage at diagnosis in over 1,200 consecutive public hospital patients in Soweto, South Africa: a case series. Breast Cancer Res. 2013;15(5):R84.

6. Carr JA, Havstad S, Zarbo RJ, Divine G, Mackowiak P, Velanovich V. The association of HER-2/neu amplification with breast cancer recurrence. Archives of Surgery. 2000;135(12):1469-74.

7. Papazisis K, Habeshaw T, Miles D. Safety and efficacy of the combination of trastuzumab with docetaxel for HER2-positive women with advanced breast cancer. A review of the existing clinical trials and results of the expanded access programme in the UK. Int J Clin Pract. 2004;58(6):581-6.

8. Pao W, Miller VA. Epidermal growth factor receptor mutations, small-molecule kinase inhibitors, and non-smallcell lung cancer: current knowledge and future directions. $\mathrm{J}$ Clin Oncol. 2005 Apr 10;23(11):2556-68.

9. Ross JS, Fletcher JA, Linette GP, Stec J, Clark E, Ayers M, et al. The Her-2/neu gene and protein in breast cancer 2003: biomarker and target of therapy. Oncologist. 2003;8(4):307-25.

10. Osborne C, Wilson P, Tripathy D. Oncogenes and tumor suppressor genes in breast cancer: potential diagnostic and therapeutic applications. Oncologist. 2004;9(4):361-77.

11. Järvinen TA, Tanner $M$, Rantanen $V$, Bärlund M, Borg $\AA$, Grénman S, et al. Amplification and deletion of topoisomerase II associate with ErbB-2 amplification and affect sensitivity to topoisomerase II inhibitor doxorubicin in breast cancer. The American journal of pathology. 2000;156(3):839-47.

12. Landaverde D, Verma S. Recent treatment advances in HER2-positive metastatic breast cancer: a clinical approach. Clinical Practice. 2012;9(3):287-99.

13. Extra JM, Antoine EC, VincentSalomon A, Delozier T, Kerbrat P, 
Bethune-Volters A, et al. Efficacy of trastuzumab in routine clinical practice and after progression for metastatic breast cancer patients: the observational Hermine study. Oncologist. 2010;15(8):799-809.

14. Vogel CL, Cobleigh MA, Tripathy D, Gutheil JC, Harris LN, Fehrenbacher L, et al. Efficacy and safety of trastuzumab as a single agent in first-line treatment of HER2-overexpressing metastatic breast cancer. J Clin Oncol. 2002 Feb 1;20(3):719-26.

15. Marty $M$, Cognetti $F$, Maraninchi $D$, Snyder R, Mauriac L, Tubiana-Hulin M, et al. Randomized phase II trial of the efficacy and safety of trastuzumab combined with docetaxel in patients with human epidermal growth factor receptor 2-positive metastatic breast cancer administered as first-line treatment: the M77001 study group. J Clin Oncol. 2005 Jul 1;23(19):4265-74.

16. Milani A, Montemurro F, Gioeni L, Aglietta M, Valabrega G. Role of trastuzumab in the management of HER2-positive metastatic breast cancer. Breast Cancer (Dove Med Press). 2010 Nov 24;2:93-109.

17. Caldarella A, Crocetti E, Bianchi S, Vezzosi V, Urso C, Biancalani M, et al. Female breast cancer status according to ER, PR and HER 2 expression: a population based analysis. Pathology \& Oncology Research. 2011;17(3):753-8.

18. Singh R, Gupta S, Pawar SB, Pawar RS, Gandham SV, Prabhudesai S. Evaluation of ER, PR and HER-2 receptor expression in breast cancer patients presenting to a semi urban cancer centre in Western India. J Cancer Res Ther. 2014 Jan-Mar;10(1):26-8.

19. Vaz-Luis I, Winer EP, Lin NU. Human epidermal growth factor receptor-2positive breast cancer: does estrogen receptor status define two distinct subtypes? Ann Oncol. 2013 Feb;24(2):28391.
20. Puig-Vives M, Sanchez M, SanchezCantalejo J, Torrella-Ramos A, Martos C, Ardanaz E, et al. Distribution and prognosis of molecular breast cancer subtypes defined by immunohistochemical biomarkers in a Spanish populationbased study. Gynecol Oncol. 2013;130(3):609-14.

21. Telli M, Kurian A, Chang E, Keegan T, Ford J, Gomez S. Asian race and breast cancer subtypes: a study from the California Cancer Registry. ASCO Annual Meeting Proceedings; ; 2008.

22. Su Y, Zheng $\mathrm{Y}$, Zheng $\mathrm{W}, \mathrm{Gu} \mathrm{K}$, Chen $\mathrm{Z}, \mathrm{Li} \mathrm{G}$, et al. Distinct distribution and prognostic significance of molecular subtypes of breast cancer in Chinese women: a population-based cohort study. BMC Cancer. 2011;11(1):1.

23. Vici P, Pizzuti L, Natoli C, Gamucci T, Di Lauro L, Barba M, et al. Triple positive breast cancer: a distinct subtype? Cancer Treat Rev. 2015;41(2):69-76.

24. Kennecke H, Yerushalmi R, Woods R, Cheang MC, Voduc D, Speers CH, et al. Metastatic behavior of breast cancer subtypes. J Clin Oncol. 2010 Jul 10;28(20):3271-7.

25. Song Q, Huang R, Li J, Fan J, Zheng S, Zhang $\mathrm{B}$, et al. The diverse distribution of risk factors between breast cancer subtypes of ER, PR and HER2: a 10year retrospective multi-center study in China. PloS one. 2013;8(8):e72175.

26. Gaedcke J, Traub F, Milde S, Wilkens L, Stan A, Ostertag H, et al. Predominance of the basal type and HER-2/neu type in brain metastasis from breast cancer. Modern pathology. 2007;20(8):864-70.

27. Clayton AJ, Danson S, Jolly S, Ryder WDJ, Burt PA, Stewart AL, et al. Incidence of cerebral metastases in patients treated with trastuzumab for metastatic breast cancer. $\mathrm{Br} \mathrm{J}$ Cancer. 2004;91(4):639-43. 
28. Láng I, Bell R, Feng F, Lopez R, Jassem J, Semiglazov V, et al. Trastuzumab retreatment after relapse on adjuvant trastuzumab therapy for human epidermal growth factor receptor 2-positive breast cancer: Final results of the Retreatment after HErceptin Adjuvant trial. Clin Oncol. 2014;26(2):81-9.

29. Vicente C, Serrano N, Agustín MJ, Alonso V, Palomo P, Huarte R. Cardiotoxicidad asociada a trastuzumab en la práctica clínica asistencial. Farmacia Hospitalaria. 2009;33(4):202-7.

30. Guarneri V, Lenihan DJ, Valero V, Durand JB, Broglio K, Hess KR, et al. Long-term cardiac tolerability of trastuzumab in metastatic breast cancer: the M.D. Anderson Cancer Center experience. J Clin Oncol. 2006 Sep 1;24(25):4107-15.

31. Perez EA. Cardiac toxicity of ErbB2targeted therapies: what do we know? Clinical breast cancer. 2008;8:S114-20.

32. Fedele C, Riccio G, Malara AE, D’Alessio G, De Lorenzo C. Mechanisms of cardiotoxicity associated with ErbB2 inhibitors. Breast Cancer Res Treat. 2012;134(2):595-602.

33. Tsai $\mathrm{H}$, Isaacs $\mathrm{C}, \mathrm{Fu} \mathrm{A}$, Warren $\mathrm{J}$, Freedman A, Barac A, et al. Risk of cardiovascular adverse events from

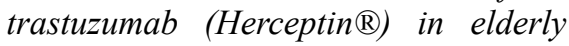
persons with breast cancer: a population-based study. Breast Cancer Res Treat. 2014;144(1):163-70.

\section{CONFLICTO DE INTERÉS Y/O AGRADECIMIENTOS}

Los autores declaran que no existió ningún conflicto de interés en el presente reporte.

\section{ANEXOS}




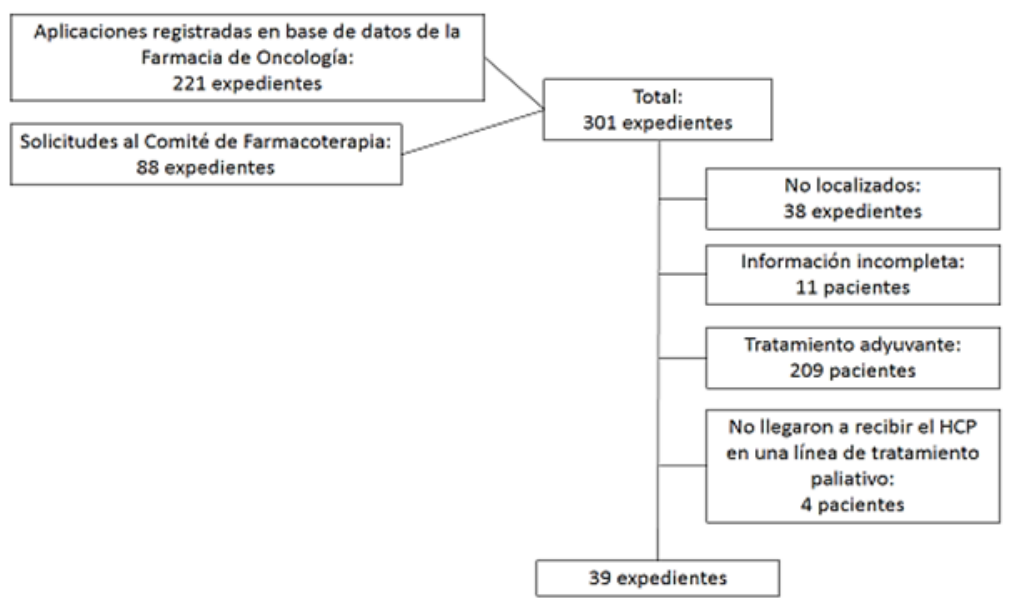

Gráfico No.1. Diagrama de Concentración

Fuente: Propia

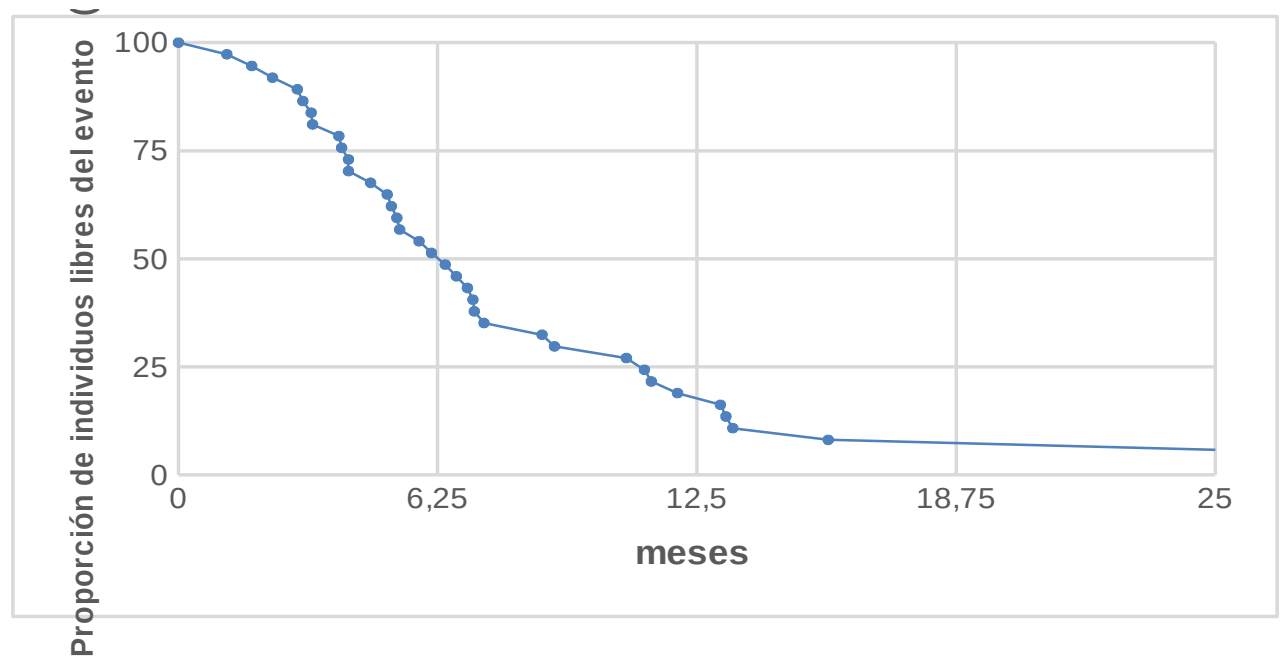

Gráfico No.2. Supervivencia Libre de Progresión en meses

Fuente: Propia

Gráfico No.3. Supervivencia general según Kaplan Meier, mediana 33.5 meses (IC 95\%: 22.1-44.8)

Fuente: Propia 
Gráfico No.4 Probabilidad de

supervivencia según estado hormonal

Fuente: Propia

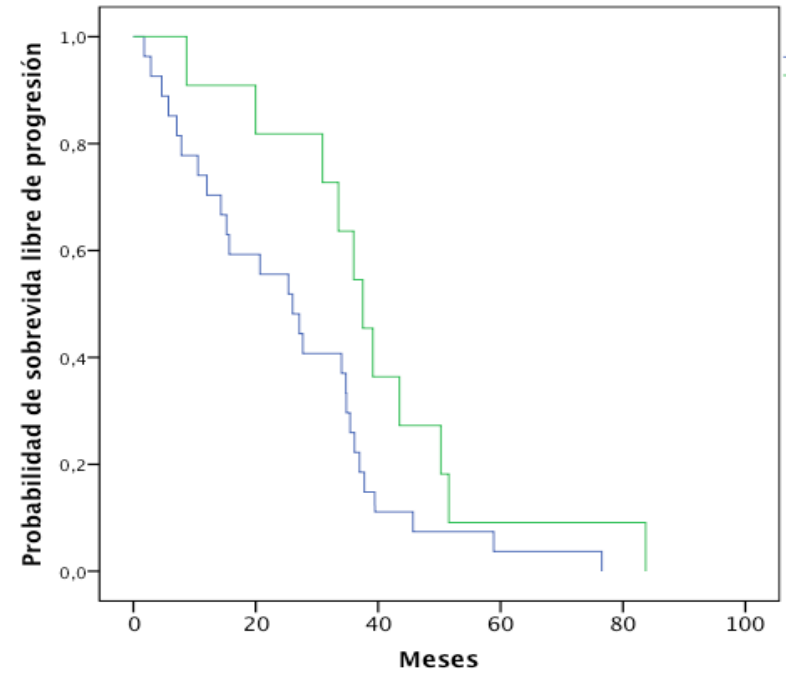

38

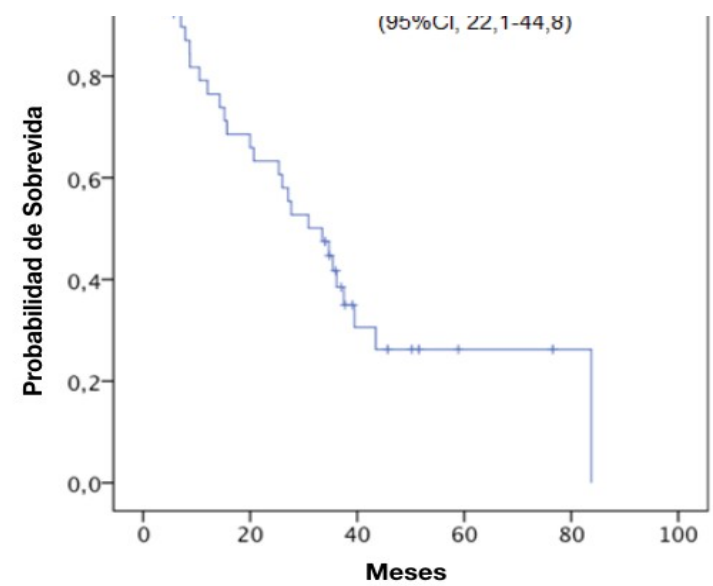

racterísticas Poblacionales

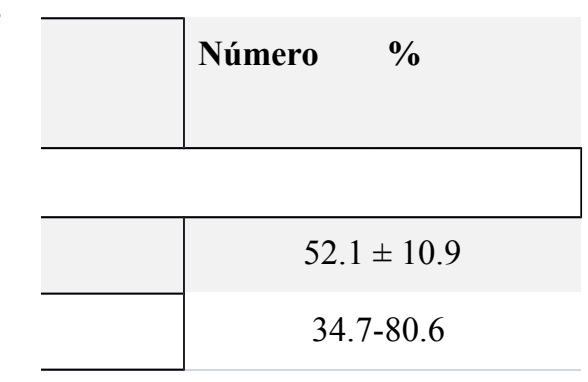

$\underline{r}$ agosto 2017 


\begin{tabular}{|c|c|c|}
\hline \multicolumn{3}{|l|}{ Estado Menopáusico } \\
\hline Premenopausia & 20 & 48 \\
\hline Menopausia & 21 & 52 \\
\hline \multicolumn{3}{|l|}{ Terapias Previas } \\
\hline Tratamiento hormonal (adyuvante, paliativo o ambos) & 15 & 35 \\
\hline Radioterapia (adyuvante, paliativa o ambas) & 23 & 56 \\
\hline Adyuvancia & 20 & 46 \\
\hline Neoadyuvancia & 10 & 23 \\
\hline Periodo libre de enfermedad (mediana en meses) & \multicolumn{2}{|c|}{19.3} \\
\hline \multicolumn{3}{|l|}{ Estado Ganglionar } \\
\hline No & 7 & 16 \\
\hline $\mathrm{N}$ positivo o desconocido & 36 & 84 \\
\hline Densidad ganglionar $\pm \mathrm{DE}$ & \multicolumn{2}{|c|}{$0.57 \pm 0.38$} \\
\hline \multicolumn{3}{|l|}{ Sitios de Metástasis } \\
\hline Hueso & 25 & 30 \\
\hline Extraóseas & 59 & 70 \\
\hline
\end{tabular}

Fuente: Propia 